\title{
Qualitative Simulation Research on Public Behavior of Sudden Power Disaster based on QSIM Theory
}

\author{
Ding-Hua ZHANG ${ }^{1, a,{ }^{*}}$, Yun-Ru KONG ${ }^{1, b}$, Liang CHENG $^{1, c}$ \\ ${ }^{1}$ Public administration, South China University of Technology, Guangzhou, China \\ adhzhang@scut.edu.cn, b404450719@qq.com, ccllucky@126.com
}

*Corresponding author

\begin{abstract}
Keywords: QSIM theory, PSR framework, Public behavior, Collective behavior, Qualitative simulation.
\end{abstract}

\begin{abstract}
In the sudden electricity disaster, as the disaster bearing body, if the public take inappropriate behavior decision-making, will aggravate the social chaos, so that the public behavior of emergency management is imperative. Using the qualitative analysis method of QSIM, this paper analyzes the qualitative evolution of public behavior and its influence factors, and simulates evolution paths of public cluster behavior, according to the relationship between environmental factors and government decision-making factors and four kinds of public behavior interaction. The conclusion of the qualitative analysis of public behavior is as follows: under the dual influence of the environment and the government, the state of public behaviors in power disasters is from no special reaction to save itself, and then turned to complaints and cluster as time goes on. While cluster intentions of leaders, participants, followers are the precondition of collective behavior's formation, and the state of isolates and followers will affect the following cluster degree.
\end{abstract}

\section{Introduction}

Sudden power disaster mainly refers to large area long-term blackouts, due to regional power grids, provincial power grids and urban power grid to reduce the load that caused by natural disasters, external damage and power safety accidents and other reasons, which can affect and threaten the national security, social stability and the people's production and life [1].With the popularization of power system, power has become an important energy for the public to survive. Once the sudden power disaster comes, the public, as a direct disaster victim, will take certain measures to deal with sustained blackouts in large areas and frequent secondary events, some of which, such as irrational cluster behaviors, will hinder the government's efforts in disaster relief and reconstruction, exacerbate social chaos and threaten social stability. Whether it is for the maintenance of social stability of the governance needs, or the responsibility to serve the people, we should pay attention to the public action in the sudden power disaster. Through making us understand the evolution of public behavior, qualitative simulation of public behavior can provide advice and help for government's emergency management after the disaster, especially for the decision-making of public behavior.

Qualitative simulation is a kind of reasoning method that uses non digital means to process information input, modeling, behavior analysis and output simulation, and qualitatively deduce the qualitative behavior of the system [2]. QSIM algorithm is one of the methods of qualitative simulation, which describe mock objects by using qualitative differential equation to describe the object of qualitative differential equations consisting of variables and constraints, and is a method of conjecturing the variation trend of the system by starting from the initial state of qualitative variables, making inference according to certain rules, and getting the direction and magnitude of the change in the variable, under qualitative constraints[3]. Therefore, this paper studies qualitative simulation of public behavior based on the QSIM algorithm, which contains the simulation analysis of the evolution of public behavior under the influence of many factors in sudden power disasters, and the simulation analysis of the development paths of cluster behavior with the interaction 
between four types of behavior subjects within a group, and the combination of environmental factors and government factors.

\section{Qualitative Simulation of Public Behavior and Influencing Factors}

\section{Qualitative Analysis of Public Behavior and Its Influencing Factors in PSR Framework}

PSR is "pressure-state-response" model, mainly used in environmental analysis, that describes and analyzes the various influencing factors in the field of environment and the relationship between them, and present an internal conflict and seek ways to resolve conflicts [4]. Under the background of sudden power disaster, the public behaviors and their influencing factors are analyzed by using the PSR framework, and the detailed analysis framework is shown in Fig.1.

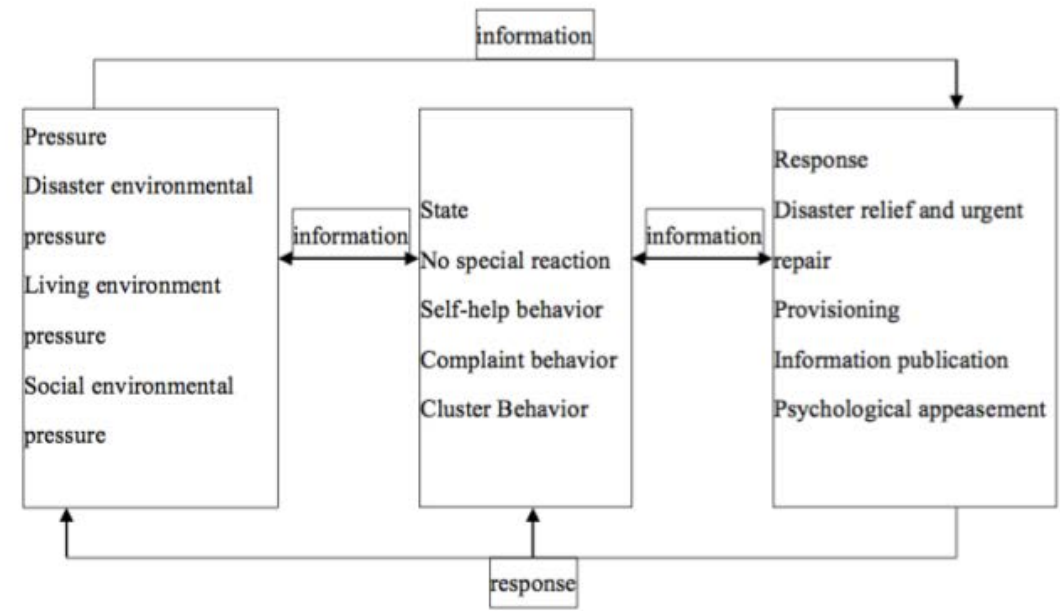

Fig.1 PSR analytical framework

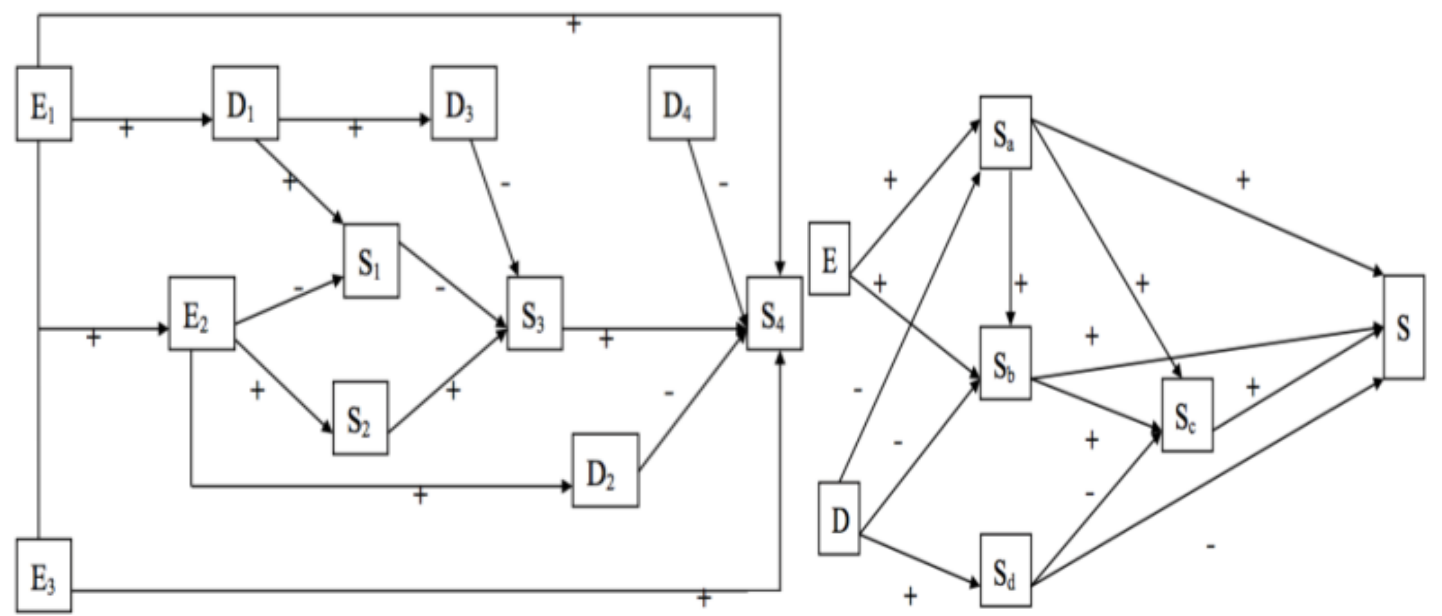

Fig. 2 The causal model

Fig. 3 The causal model

In the analytical framework, pressure factors refer to environmental pressures that exert psychological pressure on the public and change public behaviors in the sudden power disaster. The environmental pressures mainly show in three aspects. The first one is the disaster environment pressure refers to the pressure of large area blackouts for a long time. The second one is the living environment pressure. If the power supply is interrupted for a long time, water, gas, traffic and communications will be also interrupted, which gradually increase the pressure on public life and survival, as the disaster continues. The third one is the social environment pressure. Individual behavior changes because of the stress from others' behavior choices and the social environment in the sudden power disaster. Status factors are public's behavior decisions, which can be divided into 
4 types: no special reaction, self-help, complaint and cluster, according to rank and influence [5]. The response factor refers to a series of measures taken by the government to deal with the blackout and secondary effects, reduce he social loss and the burden of the public in sudden power disasters. These measures include four main dimensions: disaster relief and rush-repairs, provisioning, information distribution, and psychological appeasement.

According to the framework of PRS, after the sudden power disaster, the growth of environmental stress factors will affect status factors, so that the public behavior will take on a certain state with growing pressure. Then changes in pressure and state will make the governments take some decisions as response to environmental pressures and public behaviors. Altered environmental stress conditions in response to the impact of environmental pressure, and government decision-making which directly acting on the public, will affect and change the response and status of public behavior again.

\section{Variable Determination}

Combined with PSR framework and QSIM algorithm, the environmental pressure, the government decision-making response and the public behavior are three qualitative variables, which are set as environmental variable $\mathrm{E}$, decision variable $\mathrm{D}$ and state variable $\mathrm{S}$ respectively. The environmental variable $\mathrm{E}$ and the decision variable $\mathrm{D}$ determine the state variable $\mathrm{S}$. As environmental variable, pressures of disaster environment, pressures of social environment, pressures of living environment, can be expressed as $\mathrm{E}=\left\{\mathrm{E}_{1}, \mathrm{E}_{2}, \mathrm{E}_{3}\right\}$. Disaster relief and rush-repairs, provisioning, information distribution, psychological appeasement, as decision variables, can be expressed as $D=\left\{D_{1}, D_{2}, D_{3}\right.$, $\left.\mathrm{D}_{4}\right\}$. And no special the reaction, self-help behavior, complaint behavior, cluster behavior, as the state variables, can be expressed as: $S=\left\{S_{1}, S_{2}, S_{3}, S_{4}\right\}$. The specific causal relationship between these variables can be represented by the model in Fig.2. In the Fig. 2, the arrow is used to connect the variables, indicating the causal relationship and direction of the variables. "-" means the two variables are negatively correlated, and "+" means positive correlation [6].

In the QSIM algorithm, the state of the qualitative variable QS (f,t)or QS (f,ti,ti+1) at the significant time points $t_{i}$, or time periods $\left(t_{i}, t_{i}+1\right)(i=1,2,3,4,5 \ldots n)$ is represented by two tuples $<$ qval, qdir $>$, in which qval is a qualitative value representing the degree of the state of the qualitative variable, and qdir is the direction of the change in the state of the variable. In this paper, the values of qval are set to $\{-2,-1,0,1,2\}$, respectively representing that the degree of environmental pressure, government decision-making response and public behavior state is very low, low, general, high and very high. At the same time, make the value of qdiras $\{-, 0,+\}$, respectively representing that directions for changes of qualitative variables in the future are downward, stable and upward [7].

\section{Determination of the Rules for Transformation and Filtering.}

When using QSIM to simulate the change of qualitative variables, the conversion rules $\mathrm{P}$ and I must be observed in order to ensure the correctness of the reasoning. Conversion rules $\mathrm{P}$ should be ordered, when it's time to reason qualitative variables from time points to time periods. And conversion rules I should be ordered when it's time to reason qualitative variables from time periods to time points. [8]

Qualitative state set, obtained from following transition rules P and I, isn't the final results of qualitative simulation, but is only all possible states of mechanical reasoning. It also needs reasonable filtration to make it rational, on the basis of causality constraint filtering and matching consistency filtering. The first principles are maximum and minimum [9], where the state of qualitative variables can't exceed the bounds of the specified maximum and minimum. The provisions of the maximum and minimum values of qualitative variables are 2 and -2, which means that when the qualitative state value is 2, the next state can't have upward tendency and the direction cannot be "+"; and when the qualitative value is -2 , the direction of change in next state can only be "+" or "0", and won't show a downward trend. Then, the environmental pressure, the response of government decision-making and the public behavior, are all processes of continuous development, which means that the conversion of the state also has certain continuity, and there won't be 
salutatory subsequent status. Finally, when a variable has several dependent variables, it should be combined with actual situation to judge the strength of each dependent variable over time, and determine the status of the variable.

\section{Process of Qualitative Reasoning.}

This paper will follow the basic steps of qualitative simulation in the three time scenarios of $\left[\mathrm{t}_{0}, \mathrm{t}_{1}\right.$, $t_{2}$ ], and simulate the changes of influencing factor and public behavior states in the context of sudden power disaster.

First, the states of the variable in $t_{0}$ are taken as the initial states, and their qualitative values and the directions of change are specified respectively. At the initial time point, $\mathrm{QS}\left(\mathrm{E}_{1}, \mathrm{t}_{0}\right)=<1,0>$, $\mathrm{QS}\left(\mathrm{E}_{2}, \mathrm{t}_{0}\right)=<0,0>, \mathrm{QS}\left(\mathrm{E}_{3}, \mathrm{t}_{0}\right)=<0,0>$; $\mathrm{QS}\left(\mathrm{D}_{1}, \mathrm{t}_{0}\right)=<0,0>, \mathrm{QS}\left(\mathrm{D}_{2}, \mathrm{t}_{0}\right)=<0,0>, \mathrm{QS}\left(\mathrm{D}_{3}, \mathrm{t}_{0}\right)=<0,0>$, $\mathrm{QS}\left(\mathrm{D}_{4}, \mathrm{t}_{0}\right)=<0,0>$; $\mathrm{QS}\left(\mathrm{S}_{1}, \mathrm{t}_{0}\right)=<2,->, \mathrm{QS}\left(\mathrm{S}_{2}, \mathrm{t}_{0}\right)=<0,0>, \mathrm{QS}\left(\mathrm{S}_{3}, \mathrm{t}_{0}\right)=<0,0>, \mathrm{QS}\left(\mathrm{S}_{4}, \mathrm{t}_{0}\right)=<0$, $0>$.Among these factors, behavior of no special reaction can only exist in early stage of sudden electric disaster, and its possibility can only decreases with the duration of disasters. Therefore, make the qualitative value as " 2 ", and the direction as “-”, in the initial state. The reason why taking the qualitative value of A as " 1 " different from the other two categories is that only when a certain pressure has been formed can the power outages and other disaster phenomenon affect the public life and social atmosphere, and then pressures of living environment and pressures of social environment appear. After the initial states are determined, it is time to reason the states at the time and time points based on the rules of PI in turns, and interpret and filter all possible subsequent states. And the final simplified results of simulation are shown in table 1.

Table 1 Evolution of public behaviors after disaster

\begin{tabular}{|l|l|l|l|l|l|}
\hline \multirow{2}{*}{$\begin{array}{c}\text { Qualitative } \\
\text { variable }\end{array}$} & \multicolumn{5}{|c|}{ time } \\
\cline { 2 - 6 } & $\mathrm{t}_{0}$ & $\left(\mathrm{t}_{0}, \mathrm{t}_{1}\right)$ & $\mathrm{t}_{1}$ & $\left(\mathrm{t}_{1}, \mathrm{t}_{2}\right)$ & $\mathrm{t}_{2}$ \\
\hline $\mathrm{E}_{1}$ & $<1,0>$ & $<(1,2),+>$ & $<2,0>$ & $<(1,2),->$ & $<1,->$ \\
\hline $\mathrm{E}_{2}$ & $<0,0>$ & $<(0,1),+>$ & $<1,+>$ & $<(1,2),+>$ & $<2,0>$ \\
\hline $\mathrm{E}_{3}$ & $<0,0>$ & $<(0,1),+>$ & $<1,+>$ & $<(1,2),+>$ & $<2,0>$ \\
\hline $\mathrm{D}_{1}$ & $<0,0>$ & $<(0,1),+>$ & $<1,+>$ & $<(1,2),+>$ & $<2,0>$ \\
\hline $\mathrm{D}_{2}$ & $<0,0>$ & $<(0,1),+>$ & $<1,+>$ & $<(1,2),+>$ & $<2,0>$ \\
\hline $\mathrm{D}_{3}$ & $<0,0>$ & $<(0,1),+>$ & $<1,+>$ & $<(1,2),+>$ & $<2,0>$ \\
\hline $\mathrm{D}_{4}$ & $<0,0>$ & $<0,0>$ & $<0,0>$ & $<(0,1),+>$ & $<1,+>$ \\
\hline $\mathrm{S}_{1}$ & $<2,->$ & $<(1,2),->$ & $<1,->$ & $<(0,1),->$ & $<0,->$ \\
\hline $\mathrm{S}_{2}$ & $<0,0>$ & $<(0,1),+>$ & $<1,0>$ & $<(0,1),->$ & $<0,->$ \\
\hline $\mathrm{S}_{3}$ & $<0,0>$ & $<(0,1),+>$ & $<1,+>$ & $<(1,2),+>$ & $<2,0>$ \\
\hline $\mathrm{S}_{4}$ & $<0,0>$ & $<(0,1),+>$ & $<1,+>$ & $<(1,2),+>$ & $<2,0>$ \\
\hline
\end{tabular}

From simulations of the public behavior and influence factors of post disaster in three environmental variables, pressure of disaster environment has a process that it increases first and then decreases with time passes. And its impact on the public behavior gradually reduced, while the pressures of social environment and living environment continued to increase gradually with pressure disasters, which means with the occurrence and development of power disaster, stress of environment continues to strengthen. In the decision variables, the response degree of government decision-making shows increasing trend, but various factors have different initial states and development speeds, which is closely related to the changes of environment variables' pressure at each stage of the disaster. They also have same trend. In other words, the strength of the government response' s pressures have causality with the increasing pressures of social environment and living environment. In the state of public behavior, as time continues, the number of public of no special reaction and self-help is from high to low, and the number of people with complaints and collective behavior tendency is gradually increased. The emergence of this phenomenon is the public dynamic response to the influence of strong pressure and government decision-making variables. In general, in the early days of sudden power disasters, there are negative impacts on public accustomed to using electricity when a large area of power failure happens. The environmental pressure began to increase, and the government response lags behind the sudden 
blackouts, so the degree of government response is on general level. with the general view of power outages, the public often do not appear special reaction, but wait for the government to solve the blackouts. As time pass, the government responses to environmental pressure can't be offset, the public start to help themselves to solve the need for survival, and complaints and cluster tendency begin to increase. while environmental pressures continue to increase, and the degree of response of government decisions begin to improve. When the time goes into $t_{2}$, the overall environmental pressure reaches the maximum, and the degree of government response is on the highest level, the self-help reduces, and the complaint and cluster reaches the peak.

\section{Simulation of the Evolution Path of Public Cluster Behavior}

\section{Causality in the Evolution of Cluster Behavior}

With the deterioration of the environment, gradually increased environmental pressure, the level of government decision-making is also rising. When the government response can't offset the environmental pressure, the number of people choosing clusters in the public begin to increase, and the degree of the cluster state of public behavior increases. When the ambient pressure is the strongest, and the government's responses have reached the most comprehensive level, which means the functions of these two factors are on the equilibrium state, the status of public cluster reaches its maximum. In this process, the selection processes of different characteristics and types of public cluster behaviors are different. According to Kurt Lewin's theory of group dynamics [10], it is not only affected by environmental factors and government decision-making factors, but also influenced by other types of public. That is to say, the cluster behavior of the public form finally after the disaster, which is not the result of a simple superposition of public individual behaviors, but the product of the interaction of public individual behaviors influenced by the environmental factors and the government decision-making factors. In the context of sudden electric disaster, the public in cluster behavior can be divided into four types. The first type is the leaders who play a role of organization and leadership in the cluster behavior, influencing the direction of the behaviors of the whole group. They have the high cluster willingness and will incite public gathering into groups and organizations to take cluster action. The second type is participants are activists in group activities, with high enthusiasm of the cluster and low immunity to rumors and agitation; the third type is the followers, having general cluster willingness and weak independence, who are vulnerable to the influence of the community environment and will generate following behavior under group pressure. The fourth type is isolated whose autonomy of individual behavior can be maintained without being influenced by the group, under the background of sudden electric disaster. Generally, they have a strong ability to withstand disasters, a high immunity to the environment, and a high level of trust in the government. What's more, they have a negative effect on the behavior of the cluster.

Make the environmental variables $\mathrm{E}$ to represent environmental pressures, and the decision variable D to represent the government's emergency policy and emergency action, and Sa, Sb, Sc, Sdto represent the behavioral capabilities of leaders, participants, followers, and isolated individuals, and $\mathrm{S}$ to represent the degree of cluster behavior. The causalities between E, D, Sa, Sb, Sc, Sd are shown in the Fig.3.E and D are causal variables, $\mathrm{S}$ is the result variable, and $\mathrm{Sa}, \mathrm{Sb}$, Sc, and Sd are both causal variables and outcome variables. Specifically, under the background of sudden electric disaster, environmental factors $\mathrm{E}$ and government decision-making factors $\mathrm{D}$ have a common influence on the leaders and participants: malignant disaster environment will increase the appeal of the leaders and the participation of the participants, and the government takes an active role in coping with decision-making and behavior, which have the opposite effect to the behavior ability of leaders and participants. And the improvement of leader's leadership will improve participants' participation. Isolated people are not affected by environmental factors, but active government decision-making will enhance the independence of isolated people. The followers' capacities for action are positively related to the leaders' and participants' capacities for action, and are negatively related to the isolated person. Finally, cluster status is determined jointly by Sa, Sb, Sc and Sd. 


\section{Qualitative Reasoning of Cluster Behavior}

According to the QSIM algorithm, the values of environmental variables and decision variables are set to $\{-, 0,+\}$, representing three grades, which are low, generally, high grade, of the extent of the environment and the governments' emergency responses. The values of state variables $S_{a}, S_{b}, S_{c}, S_{d}$, are set to $\{-1,0,1\}$, respectively indicating leadership, cluster following and agglomeration degree are very low, or in general, or very high, and the directions of state variables are $\{-, 0,+\}$, representing the decreasing, sustaining, rising. Qualitative reasoning about cluster behavior is carried out mainly in time $\left[\mathrm{t}_{0},\left(\mathrm{t}_{0}, \mathrm{t}_{1}\right), \mathrm{t}_{1}\right]$.

At the initial time $t_{0}$, make QS $\left(e, t_{0}\right)=<+>$, QS $\left(d, t_{0}\right)=<+>$, according to the conditions of the cluster occurrence, Which means that the environment is deteriorating and the government is strengthening works of rescue and recovery, in the wake of the sudden electric disaster. The initial statuses of other state variables associated with the public are set as: $\mathrm{QS}\left(\mathrm{S}_{\mathrm{a}}, \mathrm{t}_{0}\right)=<0,0>, \mathrm{QS}\left(\mathrm{S}_{\mathrm{b}}\right.$, $\left.\mathrm{t}_{0}\right)=<0,0>, \mathrm{QS}\left(\mathrm{S}_{\mathrm{c}}, \mathrm{t}_{0}\right)=<0,0>, \mathrm{QS}\left(\mathrm{S}_{\mathrm{d}}, \mathrm{t}_{0}\right)=<0,0>\mathrm{QS}\left(\mathrm{S}, \mathrm{t}_{0}\right)=<0,0>$.According to the state transition rules of $\mathrm{P}$ and $\mathrm{I}$, the following status of each variable can be got from reasoning and filtration of explanation. And when $\mathrm{t}=\mathrm{t}_{1}$, the status of each state variable is as follows. $\mathrm{Sa}: \mathrm{I}_{2}=>\mathrm{QS}<1,0>$, $\mathrm{I}_{1}=>\mathrm{QS}<0,0>; \mathrm{S}_{\mathrm{b}}: \mathrm{I}_{2}=>\mathrm{QS}<1,0>, \mathrm{I}_{1}=>\mathrm{QS}<0,0>; \mathrm{S}_{\mathrm{d}}: \mathrm{I}_{1}=>\mathrm{QS}<0,0>, \mathrm{I}_{4}=>\mathrm{QS}<(1,0),+>; \mathrm{S}_{\mathrm{c}}: \mathrm{I}_{2}=>\mathrm{QS}<$ $1,0>, \mathrm{I}_{4}=>\mathrm{QS}<(0,1),+>, \mathrm{I}_{1}=>\mathrm{QS}<0,0>, \mathrm{I}_{7}=>\mathrm{QS}<(-1,0),->$; $\mathrm{S}: \mathrm{I}_{2}=>\mathrm{QS}<1,0>, \mathrm{I}_{4}=>\mathrm{QS}<(0,1),+>$, $\mathrm{I}_{1}=>\mathrm{QS}<0,0>, \mathrm{I}_{7}=>\mathrm{QS}<(-1,0),->$.

Finally, there are two kinds of states for the public cluster behavior, namely, "producing" and "not producing". The paths of public collective behavior come out after the combination and filtration these behaviors. They are shown in Table 2.

Table 2 evolutional paths of public cluster behavior after disaster

\begin{tabular}{|l|ll|ll|}
\hline & \multicolumn{2}{|l|}{ Cluster behavior } & \multicolumn{2}{l|}{ No cluster behavior } \\
\hline QS $\left(e, \mathrm{t}_{0}\right)=<+>$ & $\mathrm{I}_{2} \mathrm{I}_{2} \mathrm{I}_{2} \mathrm{I}_{1} \mathrm{I}_{2} \mathrm{I}_{2} \mathrm{I}_{2} \mathrm{I}_{4} \mathrm{I}_{1} \mathrm{I}_{2} \mathrm{I}_{2} \mathrm{I}_{2} \mathrm{I}_{1} \mathrm{I}_{4} \mathrm{I}_{2}$ & & $\mathrm{I}_{1} \mathrm{I}_{1} \mathrm{I}_{1} \mathrm{I}_{1} \mathrm{I}_{1}$ & $\mathrm{I}_{1} \mathrm{I}_{1} \mathrm{I}_{1} \mathrm{I}_{4} \mathrm{I}_{1}$ \\
QS $\left(d, \mathrm{t}_{0}\right)=<+>$ & $\mathrm{I}_{2} \mathrm{I}_{2} \mathrm{I}_{2} \mathrm{I}_{1} \mathrm{I}_{4} \mathrm{I}_{2} \mathrm{I}_{2} \mathrm{I}_{4} \mathrm{I}_{1} \mathrm{I}_{4} \mathrm{I}_{2} \mathrm{I}_{2} \mathrm{I}_{1} \mathrm{I}_{4} \mathrm{I}_{4}$ & $\mathrm{I}_{2} \mathrm{I}_{2} \mathrm{I}_{4} \mathrm{I}_{4} \mathrm{I}_{4}$ & $\mathrm{I}_{1} \mathrm{I}_{1} \mathrm{I}_{1} \mathrm{I}_{4} \mathrm{I}_{7}$ & $\mathrm{I}_{1} \mathrm{I}_{1} \mathrm{I}_{7} \mathrm{I}_{4} \mathrm{I}_{1} \mathrm{I}_{1} \mathrm{I}_{1} \mathrm{I}_{7} \mathrm{I}_{4} \mathrm{I}_{7}$ \\
\hline
\end{tabular}

Through the above analysis, we can find seven main paths for the public cluster behavior after the disaster. There are two categories of the degree of clustering which are high, and rising from general to high. Obviously, as we can know from the analysis of cluster's procreant paths, the emergences of cluster behaviors mainly depend on the increase leaders and participants, that is to say, if the number of a certain group reduces or disappears because of the reduction of external factors, in a certain process, the behavior of the cluster will not happen. In addition, the influence of isolated person and the number of followers have impact on the degree of clustering. If the influence of the isolated person is increased and the followers of the cluster reduce, the degree of the public cluster will reduce.

\section{Summary}

This paper mainly deals with two parts: the evolution of public behavior state and the reasoning of cluster behavior path. The foregoing analysis divides the public behavior into four categories. These four behaviors are the results of both environmental and governmental influences. The qualitative evolution shows that the condition of no special reaction drops from high to average and continues to reduce. The status of self-rescue gradually increases from the general state in the early stage, but reduces persistently in the middle and late stages. And complaints and cluster status change from a general state to a high state. Therefore, in the context of understanding the evolution of public behavior, the government can make decisions in response to the state of public action, while providing disaster relief and fighting calamities, in order to reduce complaints and avoid cluster behavior as much as possible. And in the analysis of the evolution paths of public behavior, leaders, participants and followers produce cluster intention together, which is the precondition of cluster behavior. When the cluster behavior is bound to occur, appropriate measures should be taken to improve the independence of the isolated population and reduce the number of the followers, which 
can play a role in reducing the high degree of the cluster behavior.

\section{Acknowledgement}

This research was financially supported by the basic business fee of Central University -- Research on public group behavior modeling and government aided decision making under the chain of adverse events in coastal cities, based on Simulation. (x2ggD2152410)

\section{References}

[1]Information on http://www.gov.cn/zhengce/content/2015-11/26/content_10352.htm. (In Chinese)

[2] Rang-Hui Li, Bin Hu. Qualitative simulation technology research on incentive process of company managers,Journal of management.2 (2005)35-38. (In Chinese)

[3] Yi-Jun Liu, Gi-Fa Gu. Simulation study of public opinion corpus behavior based on QSIM algorithm, management theory.23(2011) 86-92. (In Chinese)

$[4,6]$ Shuai Lou, et al. Simulation of water resources management decision making process based on QSIM algorithm, systems engineering.12 (2013)18-29. (In Chinese)

[5] Xu Fan, Jia-Tao Guo, Wenhui Huang. The influence and consideration of large power outages on public behavior, China Electric Power Press. 3(2016). (In Chinese)

[7,9] Chuan-Liang Jia, Hong Chi, Ying Sun, Gui-Jun Li. Simulation study of emergency handling process based on QSIM algorithm, China management science.15 (2007) 740-744. (In Chinese)

[8] Bin Hu. Qualitative simulation theory and application of group behavior, Huazhong University of Science and Technology press, Wuhan, 2006. (In Chinese)

[10] De Zhang. Organizational behavior, Higher Education Press, Beijing, 2002. (In Chinese) 